\title{
TRANSFER ORBITS IN THE RESTRICTED PROBLEM
}

\author{
Antonio Fernando Bertachini de Almeida Prado* \\ Research Engineer, Depto. of Space Mechanics and Control \\ Instituto Nacional de Pesquisas Espaciais - INPE - Brazil \\ São José dos Campos - SP - 12227-010 - Brazil \\ and \\ Roger Broucke ${ }^{+}$ \\ Professor, Depto. of Aerospace Eng. and Eng. Mechanics \\ University of Texas at Austin \\ Austin-TX-78712 - USA
}

\begin{abstract}
$\underline{\text { Abstract }}$
This paper studies transfer orbits in the planar restricted three-body problem. In particular, we are searching for orbits that can be used in two situations: a) To transfer a spacecraft from one body back to the same body (known in the literature as the Hénon's problem); b) To transfer a spacecraft from one body to the respective Lagrangian points $\mathrm{L}_{4}$ and $\mathrm{L}_{5}$. To avoid numerical problems during close approaches the global Lamaitre regularization is used. Under this model, the Hénon's problem became a Lambert's three body problem, as defined and explained later in this paper.
\end{abstract}

\section{Introduction}

To solve the problem defined above we study each situation individually. In the first situation, attention is given to the family of transfer orbits involving no more than one revolution of the spacecraft. The systems under study are the ones with more important practical applications: the Earth-Sun and the Earth-Moon systems. Five families of transfer orbits are found in the region studied and the results are plotted in terms of the true anomaly. The same plots also show the evolution of the Jacobian constant. A special effort is made to reproduce some of the previously found ${ }^{1,2}$ transfer orbits with small $\Delta \mathrm{V}$, under this improved model.

In the second situation, the problem of sending a spacecraft from the Earth to the Lagrangian points $\mathrm{L}_{4}$ and $\mathrm{L}_{5}$ (in the Sun-Earth system) is treated as a natural extension of the problem of sending a spacecraft from one body back to the same body. Two transfer orbits from the Earth to $\mathrm{L}_{4}$ and two transfer orbits from the
Earth to $\mathrm{L}_{5}$ are found. Next, the numerical integration is extended beyond the desired Lagrangian point and it is found that, for all four orbits, the spacecraft passes near the Lagrangian points $\mathrm{L}_{3}, \mathrm{~L}_{4}$ and $\mathrm{L}_{5}$ and comes back to the neighborhood of the Earth. In general, the orbits found here can be applied to:

i) Transfer a spacecraft between any two points in the group formed by the Earth and the Lagrangian points $\mathrm{L}_{3}, \mathrm{~L}_{4}, \mathrm{~L}_{5}$ (in the Earth-Sun system) with near-zero $\Delta \mathrm{V}$

ii) Make a tour to the Lagrangian points for reconnaissance purposes ${ }^{3}$ with near-zero $\Delta \mathrm{V}$ for the entire tour. The small relative velocities during the close approaches are ideal for the data acquisition phase or for a rendezvous with another spacecraft. There is also a possibility to recover the spacecraft after the tour, since it returns to the Earth's neighborhood;

iii) Build a cycler transportation system linking all the points involved or only two of them. In a system like that, a heavy spacecraft can stay in one of the orbits showed here and a small spacecraft can make a "taxi service" and rendezvous with the heavy vehicle to transport persons and/or materials to/from it, similar to what happens in the systems proposed for the Earth and the Moon ${ }^{4,5}$ or for the Earth and the Mars ${ }^{5}$.

\section{Mathematical Model and Some Properties}

The model used in all phases of this chapter is the well-known planar circular restricted three-body problem. This model assumes that two main bodies $\left(\mathrm{M}_{1}\right.$ and $\mathrm{M}_{2}$ ) are orbiting their common center of mass in

\footnotetext{
* AIAA Student Member

+ Associate Felow AIAA

Copyright (C) 1994 by th American Institute of Aeronautics and Astronautics, Inc. All right reserved.
} 
circular Keplerian orbits and a third body $\left(\mathrm{M}_{3}\right)$, with negligible mass, is orbiting these two primaries. The motion of $\mathrm{M}_{3}$ is supposed to stay in the plane of the motion of $M_{1}$ and $M_{2}$ and it is affected by both primaries, but it does not affect their motion ${ }^{6}$. The standard canonical system of units associated with this model is used. Under this model, the equations of motion are:

$$
\begin{aligned}
& \ddot{x}-2 \dot{y}=x-\frac{\partial V}{\partial x}=\frac{\partial \Omega}{\partial x} \\
& \ddot{y}+2 \dot{x}=y-\frac{\partial V}{\partial y}=\frac{\partial \Omega}{\partial y}
\end{aligned}
$$

where $\Omega$ is the pseudo-potential function given by:

$$
\Omega=\frac{1}{2}\left(\dot{\mathrm{x}}^{2}+\dot{\mathrm{y}}^{2}\right)+\frac{(1-\mu)}{\mathrm{r}_{1}}+\frac{\mu}{\mathrm{r}_{2}}
$$

One of the most important reasons why the rotating frame is more suitable to describe the motion of $M_{3}$ in the three-body problem is the existence of an invariant, that is called Jacobi integral (or energy integral). There are many ways to define the Jacobi integral and the reference system used to describe this problem (see reference 6 , pg. 449). In this paper the definitions used by Broucke 7 are followed. Under this version, the Jacobi integral is given by:

$$
J=\frac{1}{2}\left(\dot{x}^{2}+\dot{y}^{2}\right)-\Omega(x, y)=\text { Const }
$$

The equations of motion given by equations (1) are right, but they are not suitable for numerical integration in trajectories passing near one of the primaries. The reason is that the positions of both primaries are singularities in the potential V (since $r_{1}$ or $r_{2}$ goes to zero, or near zero) and the precision of the numerical integration is affected every time this situation occurs.

The solution for this problem is to use regularization, that consists of a substitution of the variables for position (x-y) and time (t) by another set of variables $\left(\omega_{1}, \omega_{2}, \tau\right)$, such that the singularities are eliminated in these new variables. For the present research the Lamaître's regularization is used.

Another important property needed in this paper is the mirror image theorem ${ }^{8}$. It is an important and useful property of the planar circular restricted threebody problem. It says that: "In the rotating coordinate system, for each trajectory defined by $\mathrm{x}(\mathrm{t}), \mathrm{y}(\mathrm{t}), \dot{\mathrm{x}}(\mathrm{t}), \dot{\mathrm{y}}(\mathrm{t})$ that is found, there is a symmetric (in relation to the " $\mathrm{x}$ " axis) trajectory defined by $x(-t),-y(-t),-\dot{x}(-t),-\dot{y}(-t) "$.

\section{$\underline{\text { Results to Transfer a Spacecraft from One Body Back }}$ to the Same Body}

The theory developed in the last few sections to solve the problem of transferring a spacecraft from one body back to the same body (called the three body Lambert's problem) can be used here to solve the Hénon's problem in the case $\mu \neq 0$. The approach used here is to solve the three body Lambert's problem with the following input data: i) The initial position for $\mathrm{M}_{3}$, that is the position of $\mathrm{M}_{2}$ at the time that $\mathrm{M}_{3}$ departs from $\mathrm{M}_{2}$; ii) The final position of $\mathrm{M}_{3}$, that is the position of $M_{2}$ at the time that $M_{3}$ arrives at $M_{2}$ and; iii) The time of flight, that is $2 \pi(\tau / \pi)=2 \tau$;

The solution of the problem (output of the three body Lambert's problem) is the desired transfer orbit (in the restricted three body context), ready to be plotted as a point in the equivalent of the Hénon's diagram ${ }^{9}$. The scheme looks very simple, but it is not so easy to implement. The difficulty arises from the fact that, to get convergence in the solution of the TPBVP involved, an accurate first guess is required for each transfer orbit considered. The first "good first guess" available is the solution of the related two-body Lambert's problem (same initial and final position and time of flight, but with $\mu=0$, using two-body celestial mechanics equations), as solved in references 1 and 2 . If $\mu$ is small (such as the Earth-Sun system, where $\mu=0.000003$ ) this first guess is good enough to get convergence in the TPBVP for transfers with transfer time between 0.4 and about 9.0 canonical units of time (0.06 to 1.43 years in the SunEarth system). For transfer orbits with transfer time greater than that and/or involving more than one revolution for $\mathrm{M}_{3}$, a method to find a more accurate first guess has to be developed. Fig. 1 shows the results obtained by the numerical simulations of the Sun-Earth system, for the range $0 \leq \tau / \pi \leq 1.43$. It is the "equivalent" of the Hénon's diagram, which means that the differences from the original diagram are the following:

i) The orbit of $\mathrm{M}_{3}$ is no longer a conic (because this is a three body problem and not a two-body problem), so the concepts of "eccentric anomaly" and 
"true anomaly" do not exist anymore. For the graphs presented in this research, we plot $\tau / \pi$, where $\tau$ is half of the transfer time in canonical units, against $\underline{v} / \pi$, where $\underline{\underline{ }}$ is defined by:

$$
\begin{aligned}
& \underline{v}=v \text { if } M_{3} \text { passes periapse at } \tau=0 \\
& \underline{v}=\pi-v \text { if } M_{3} \text { passes apoapse at } \tau=0
\end{aligned}
$$

and $v$ is half of the angle travelled by $\mathrm{M}_{2}$ during the transfer. This definition has the goal of making $v$ a "generalized true anomaly" (that becomes the "true anomaly" in the case $\mu=0$ ) and $\underline{v}$ is the generalization of the $\underline{v}$ used in reference 2 (they also become the same quantity when $\mu=0$ ). Then, $\nu$ and $\tau$ (and so $\underline{v}$ and $\tau$ ) are linearly related, since the motion of $M_{2}$ around $M_{1}$ is circular (remember that $\mathrm{M}_{3}$ has negligible mass, which means that $M_{1}$ and $M_{2}$ is a two-body system);

ii) The value of the Jacobi Constant $\mathrm{J}$ (Equation 3 ) is given for several points in the diagram, since this is an important invariant in the restricted three body problem.

To study these results in further detail, it is necessary to make an analogy between the two-body and the three body problem. It means that the name "hyperbolic orbit" (in the three body context) is given to an orbit that comes from a two-body hyperbolic orbit with the inclusion of the perturbation of the third body. The same analogy applies to an elliptic orbit and the important parameters $\left(\varepsilon, \varepsilon^{\prime}, \varepsilon^{\prime \prime}\right)$, defined by Hénon ${ }^{9}$, are used again here: $\varepsilon=+1(-1)$ if the periapse is in an abscissa positive (negative); $\varepsilon^{\prime}=+1(-1)$ if the sense of the orbit is direct (retrograde); $\varepsilon^{\prime \prime}=$ if the passage at $\tau=0$ is at periapse (apoapse).

It is important to have always in mind that these parameters refer to the two-body elliptic orbits and are applied here as a valid approximation, since $\mu$ is small.

It is possible to see in this Figure the appearance of five distinct regions, when the analogy with the twobody problem is considered. They are called Regions A to $\mathrm{E}$ and they are identified by:

i) Region A: It is composed of hyperbolic orbits and it goes from $\tau / \pi=0.0796$ (the first point that gives convergence to the TPBVP) to $\tau / \pi=0.16393$, the frontier with the Region B. They have the highest values for J (it implies that the initial impulses also have the highest values) and the shortest transfer times, as expected. The notation $(--+)$ is a short form of $\varepsilon=-1, \varepsilon^{\prime}=-1$ and $\varepsilon^{\prime \prime}=+1$.

ii) Region B: It is composed of elliptic orbits that have their periapse with a negative abscissa $(\varepsilon=-1)$, travel in a retrograde (opposite to the motion of $\mathrm{M}_{2}$ ) direction $\left(\varepsilon^{\prime}=-1\right)$ and $M_{3}$ passes periapse at $\tau=0$, the middle of the transfer $\left(\varepsilon^{\prime \prime}=+1\right)$. This region starts at $(\tau / \pi)$ $=0.16393$, the boundary with Region $\mathrm{A}$ and extends to $(\tau / \pi)=0.5$, the boundary with Region C.

iii) Region C: It is composed of elliptic orbits that have their periapse with a positive abscissa $(\varepsilon=+1)$, travel in a retrograde (opposite to the motion of $\mathrm{M}_{2}$ ) direction $\left(\varepsilon^{\prime}=-1\right)$ and $\mathrm{M}_{3}$ passes apoapse at $\tau=0$, the middle of the transfer $\left(\varepsilon^{\prime \prime}=-1\right)$. This region starts at $(\tau / \pi)$ $=0.5$, the boundary with Region B and extends to $(\tau / \pi)=$ 1.0, the boundary with Region D.

iv) Region D: It is composed of elliptic orbits that have their periapse with a positive abscissa $(\varepsilon=+1)$, travel in a direct (the same of the motion of $\mathrm{M}_{2}$ ) direction $\left(\varepsilon^{\prime}=+1\right)$ and $\mathrm{M}_{3}$ passes apoapse at $\tau=0$, the middle of the transfer $\left(\varepsilon^{\prime \prime}=-1\right)$. This region starts at $(\tau / \pi)=1.0$, the boundary with Region $C$ and extends to $(\tau / \pi)=1.1$, the last point that gives convergence for the TPBVP in this region.

v) Region E: It is composed of elliptic orbits that have their periapse with a negative abscissa $(\varepsilon=-1)$, travel in a retrograde (opposite to the motion of $\mathrm{M}_{2}$ ) direction $\left(\varepsilon^{\prime}=-1\right)$ and $M_{3}$ passes apoapse at $\tau=0$, the middle of the transfer $\left(\varepsilon^{\prime \prime}=-1\right)$. This region starts at $(\tau / \pi)$ $=1.0$ and extends to $(\tau / \pi)=1.43$, the last point that gives convergence for the TPBVP in this region.

\section{Results for the Earth-Moon System}

After that the attention is turned to the EarthMoon system. This is a case with more practical interest and sooner applications, but it is also a more difficult case due to the high value of the mass parameter $(\mu=$ 0.0121505). Fig. 2 shows the results obtained, using the same definitions and conventions used for the SunEarth system. The main difference is that the single conic approximation for the first guess works only in the range $0.0637 \leq \tau / \pi \leq 0.9072$. As a consequence, only the regions $\mathrm{A}, \mathrm{B}$ and $\mathrm{C}$ in the diagram can be found. 


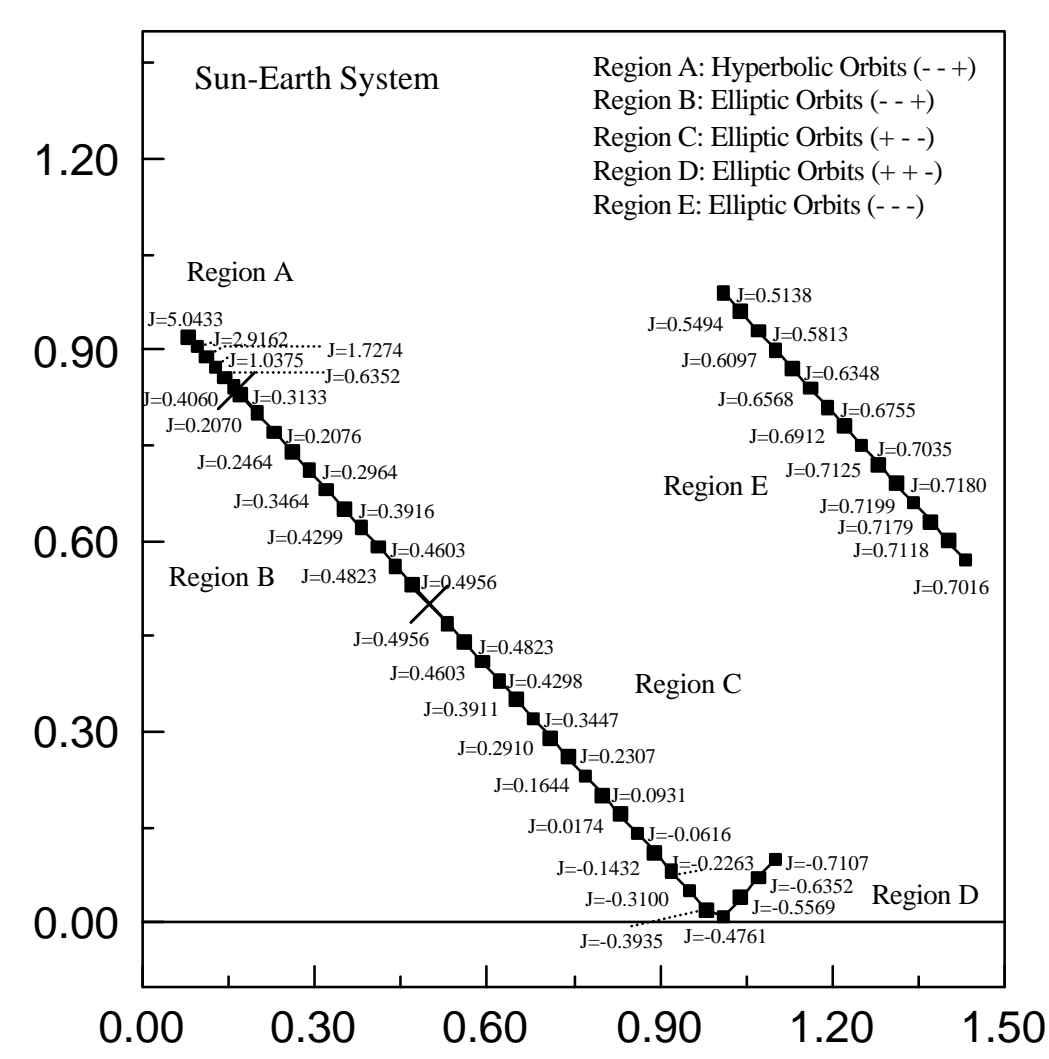

Fig. 1 - Equivalent of the Hénon's Diagram for the Sun-Earth System.

The results shown in this research do not give the whole picture of the equivalent of the Hénon's diagram $^{9}$. It shows only the portion that can be constructed by using the conic trajectory given by the case $\mu=0$ as the first guess for the Lambert's three-body problem routine. However, the method outlined here can be used to generate the whole picture, if a procedure to find a more accurate first guess can be developed.

\section{$\underline{\text { Transfer Orbits with Minimum } \Delta \mathrm{V}}$}

An important characteristic of this problem $^{1,2}$ is the family of transfer orbits with near zero $\Delta \mathrm{V}$ to transfer a spacecraft from $\mathrm{M}_{2}$ back to $\mathrm{M}_{2}$ again. These orbits, that exist in the two-body problem model (case $\mu=0$ of the three body problem), are important enough to deserve a study in the more realistic case $\mu \neq 0$. In the present section this research is performed in the SunEarth system. The two-body solution is used as the first guess and a trial and error technique (in the initial velocity) is used to find the solution. The gradient method did not work with those first guesses, due to the high nonlinear characteristic of the system in this particular case. Fig. 3 shows the trajectory, as seen in the rotating frame. It is important to note that the $\Delta \mathrm{V}$ for escape velocity from the Earth is 0.3735 canonical units (the absolute minimal for any transfer from the surface of a celestial body), which means that the $\Delta \mathrm{V}$ found in this transfer orbit (0.3839 canonical units) is only a little bit above it (0.0104 canonical units), and there is not much improvement left to be done, as far as fuel savings are concerned.

\section{Results for Transfers Between the Earth and the Lagrangian Points}

In this section, the theory developed in the first sections of this paper is used to find transfer orbits between the Earth and the Lagrangian points with minimum $\Delta \mathrm{V}$. Since the results are different for each of the trajectories studied, it is necessary to study them in detail one by one. For identification purposes the definition of the following nomenclature is made: LONG4-5 is the orbit that goes to $\mathrm{L}_{4}$ first, just after leaving the Earth, and then goes to $\mathrm{L}_{3}$ and $\mathrm{L}_{5}$, and has a long period (about 25 years); SHORT-4-5 is the orbit that visits the Lagrangian points in the same order, but with a shorter period (about 13 years); LONG-5-4 is the orbit that visits 
the Lagrangian points in a opposite order $\left(\mathrm{L}_{5}\right.$ first, and then $\mathrm{L}_{3}$ and $\mathrm{L}_{4}$ ) with a long period (about 28 years); and SHORT-5-4 is the orbit similar to LONG-5-4 (same order of points visited), but with a shorter period (about 11 years). This is the orbit with the shortest period of all the orbits studied. Each one of these orbits is described in detail in the following sections.

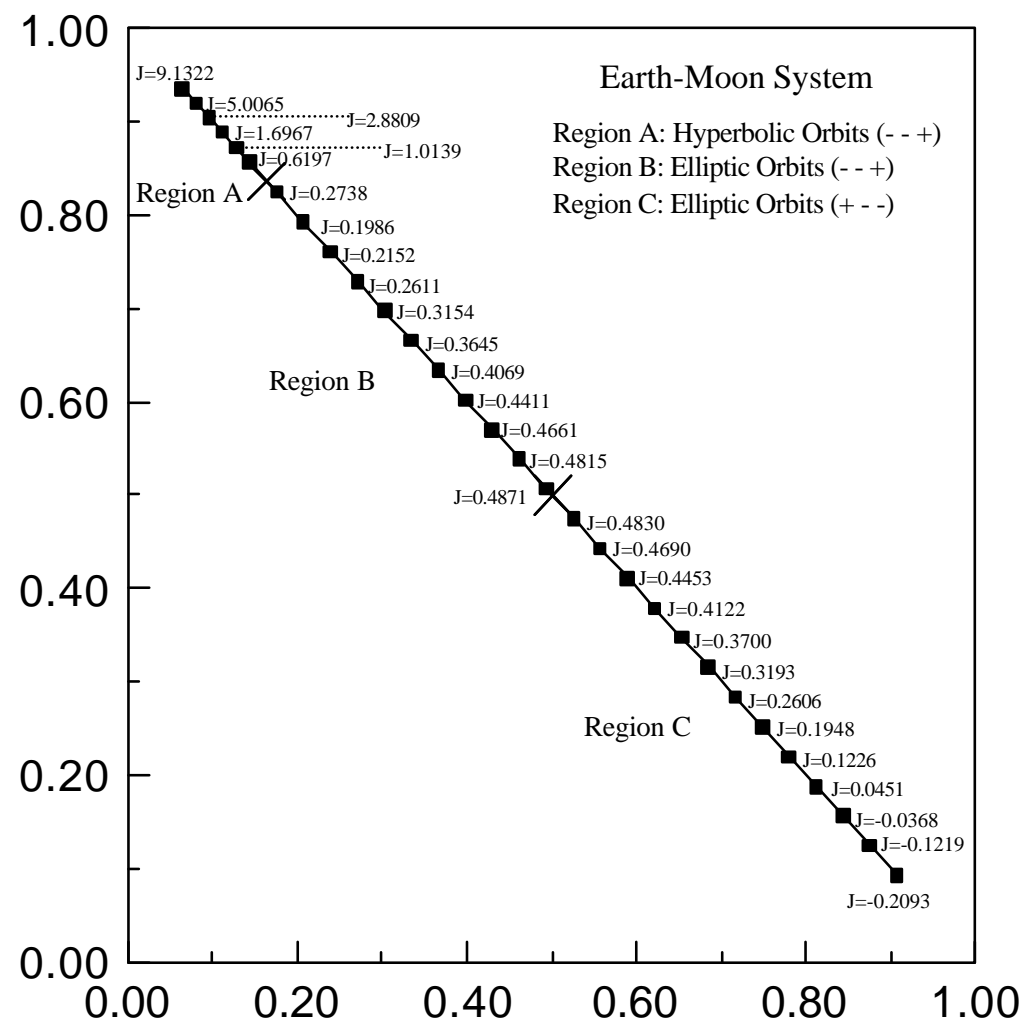

Fig. 2 - Equivalent of the Hénon's Diagram for the Earth-Moon System.

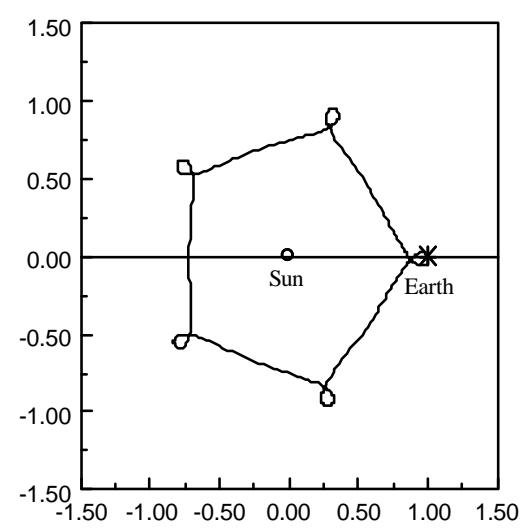

Fig. 3 - Transfer Orbit with Minimum $\Delta \mathrm{V}$ from the Earth Back to the Earth, as Seen in the Rotating Frame.

\section{The "SHORT-5-4" Orbit}

In this orbit the spacecraft $\mathrm{M}_{3}$ leaves the Earth and visits the Lagrangian points in the order $\mathrm{L}_{5}$ (in 2.12 years), $\mathrm{L}_{3}$ (in 6.46 years), $\mathrm{L}_{4}$ (in 10.88 years) and then it returns to the Earth's neighborhood (in 13.05 years). Table 1 shows the coordinates $\mathrm{x}$ and $\mathrm{y}$, the distance $\mathrm{R}$ from the Lagrangian point, the velocity components $V_{x}$ and $V_{y}$, the magnitude $\mathrm{V}$ of the velocity vector and the time $(\mathrm{t})$ lapsed from departure for the passage by all the important points, referred to the rotating frame for all orbits studied in this paper. The distances are expressed in $10^{6} \mathrm{~km}$, the velocities in $\mathrm{m} / \mathrm{s}$ and the time in years.

It is important to remember again that the $\Delta \mathrm{V}$ required for Earth's escape is $11180 \mathrm{~m} / \mathrm{s}$ (the absolute minimal for any transfer from the surface of the Earth), which means that all the $\Delta \mathrm{Vs}$ involved in Earth's escape that are shown in this research are of this order of magnitude. Then, there is not much room left for 
improvements, as far as fuel savings are concerned, in those maneuvers too. All $\Delta \mathrm{Vs}$ of this order of magnitude, when the spacecraft is leaving the Earth, or near zero, when the spacecraft is far from the Earth, are called "near-zero $\Delta \mathrm{V}$ " in this research. Fig. 4 shows the first two revolutions of this trajectory. The particular important points of this orbit are:

i) A shorter time is required for all transfers involved, when compared with the two "LONG" transfers. A period for the total tour (from the Earth back to the Earth) is about 13 years. The legs connecting $\mathrm{L}_{4}$ and $\mathrm{L}_{5}$ to the Earth has a little more than 2.1 years each;

ii) It also has closer approaches to the Lagrangian points visited, when compared to the two "LONG" transfers;

iii) After the close approach with the Earth, in the end of the first revolution, this orbit continues for a second revolution in the same direction of motion. The trajectory followed in the second revolution is not much different from the trajectory followed in the first one, and there are 12 "crossing points". Those are points that belong to the trajectory followed by the spacecraft in the first and in the second revolution. Those 12 crossing points are candidates for a one-burn maneuver that transfers the spacecraft from the trajectory it follows in the second revolution to the trajectory it follows in the first revolution. After this maneuver the spacecraft starts again its journey to $\mathrm{I}_{3}, \mathrm{~L}_{3}, \mathrm{~L}_{4}$ and the Earth. Then, a cycler transportation that links all the points involved in about 13 years is achieved.

Then, the final result is a periodic trajectory linking the Earth and the Lagrangian points $\mathrm{L}_{3}, \mathrm{~L}_{4}, \mathrm{~L}_{5}$ that has a period of about 13 years and that requires a $\Delta \mathrm{V}=0.0667(1986.7 \mathrm{~m} / \mathrm{s})$ per revolution for nominal operation.

\section{The "LONG-4-5" Orbit}

In this orbit the spacecraft $\mathrm{M}_{3}$ leaves the Earth and visits the Lagrangian points in the order $\mathrm{L}_{4}$ (in 4.69 years), $\mathrm{L}_{3}$ (in 13.96 years), $\mathrm{L}_{5}$ (in 23.29 years) and then it returns to the Earth's neighborhood (in 27.84 years). The plot of this trajectory is omitted here to save space, but it is available in reference 1 . The particular important points of this orbit are: i) It has the closest approach with the Earth at the end of the first revolution. This is an important characteristic, if a capture of the spacecraft is planned for after the tour;

ii) After this close approach, the orbit is slightly deviated by the Earth, but very close approaches to the Lagrangian points and the Earth again exist in at least two more revolutions, with no nominal corrections required. It makes this orbit the best one for a continuous cycler without nominal corrections;

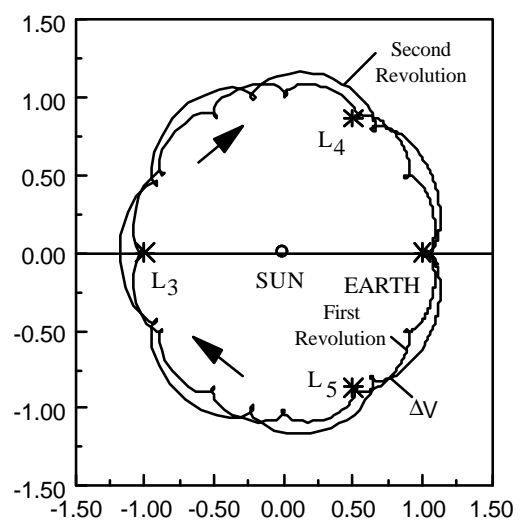

Fig. 4 - The Orbit "SHORT-5-4".

iii) This orbit has the characteristic of reversing the direction of its motion after some of the "swingbys"1,2 with the Earth. It means that some of the "swingbys" with the Earth has the effect of changing the direction of the motion in the rotating frame. During the first five revolutions of this trajectory, it occurs twice: the first one reverses the counter-clock-wise motion to a clock-wise motion at the end of the third revolution (the first three revolutions are in the counter-clock-wise direction), and the second one reverses the motion of the spacecraft to a counter-clock-wise motion again, at the end of the fourth revolution.

\section{The "SHORT-4-5" Orbit}

In this orbit the spacecraft $\mathrm{M}_{3}$ leaves the Earth and visits the Lagrangian points in the order $\mathrm{L}_{4}$ (in 1.81 years), $\mathrm{L}_{3}$ (in 5.49 years), $\mathrm{L}_{5}$ (in 9.20 years) and then it returns to the Earth's neighborhood (in 11.00 years). Fig. 5 shows the first two revolutions of this trajectory. The particular important points of this orbit are:

i) After the close approach with the Earth (in the end of the first revolution) the spacecraft starts a new tour to the Lagrangian points, in the reverse order. It 
means that the "swing-by" with the Earth has the effect of changing the direction of its motion in the rotating frame. Even more curious, integrating this trajectory for a longer time it is possible to see that the first five revolutions have alternating directions of motion. It means that there are four consecutive "swing-bys" that have the property of reversing the direction of the motion of the spacecraft. It is also noted that the second revolution has very close approaches to the Lagrangian points visited. It makes this orbit very suitable for a double-tour to the Lagrangian points, with no impulses required for nominal operation;

Table 1 - Position, Velocity and Time for the passages by the Lagrangian points in $10^{6} \mathrm{~km}, \mathrm{~m} / \mathrm{s}$ and years (referred to the Rotating frame)

\begin{tabular}{|c|c|c|c|c|c|c|c|}
\hline \multicolumn{7}{|c|}{ Orbit "SHORT-5-4" } \\
\hline Point & $\mathrm{x}$ & $\mathrm{y}$ & $\mathrm{R}$ & $\mathrm{V}_{\mathrm{x}}$ & $\mathrm{V}_{\mathrm{y}}$ & $\mathrm{V}$ & $\mathrm{t}$ \\
\hline Earth & - & - & - & 0.0 & 11130 & 11130 & 0.00 \\
\hline $\mathrm{L}_{5}$ & 74.9 & -130.1 & 0.55 & 306.8 & 590 & 664 & 2.12 \\
\hline $\mathrm{L}_{3}$ & -150.0 & 1.32 & 1.38 & 253.2 & -610 & 661 & 6.46 \\
\hline $\mathrm{L}_{4}$ & 76.1 & 129.7 & 1.30 & -128.1 & 685 & 697 & 10.88 \\
\hline Earth & 150.4 & 0.00 & 0.81 & 479.5 & 1111 & 1209 & 13.05 \\
\hline \multicolumn{7}{|c|}{ Orbit "LONG-5-4" } \\
\hline Earth & - & - & - & 0.0 & 11107 & 11107 & 0.00 \\
\hline $\mathrm{L}_{5}$ & 78.1 & -129.6 & 3.34 & -51 & -497 & 500 & 4.24 \\
\hline $\mathrm{L}_{3}$ & -153.7 & 0.0 & 4.07 & -197 & 1311 & 1337 & 12.74 \\
\hline $\mathrm{L}_{4}$ & 74.9 & 130.6 & 1.09 & 27 & 48 & 57 & 20.81 \\
\hline Earth & 149.6 & 0.7 & 0.75 & -938 & -253 & 971 & 24.88 \\
\hline \multicolumn{7}{|c|}{ Orbit "SHORT-4-5" } \\
\hline Earth & - & - & - & 0 & -11140 & 11140 & 0.00 \\
\hline $\mathrm{L}_{4}$ & 74.9 & 129.2 & 0.37 & 715 & -334 & 786 & 1.81 \\
\hline $\mathrm{L}_{3}$ & -149.3 & -0.4 & 0.46 & 18 & 789 & 792 & 5.49 \\
\hline $\mathrm{L}_{5}$ & 74.6 & -128.9 & 0.69 & -807 & 119 & 816 & 9.20 \\
\hline Earth & 149.6 & -0.1 & 0.12 & 2302 & -1346 & 2666 & 11.00 \\
\hline \multicolumn{7}{|c|}{ Orbit "LONG-4-5" } \\
\hline Earth & - & - & - & 0.0 & -11101 & 11101 & 0.00 \\
\hline $\mathrm{L}_{4}$ & 73.7 & 127.9 & 1.99 & -294.9 & 378 & 479 & 4.69 \\
\hline $\mathrm{L}_{3}$ & -144.4 & -0.1 & 5.21 & -53.6 & -1748 & 1751 & 13.96 \\
\hline $\mathrm{L}_{5}$ & 72.8 & -127.4 & 2.86 & 512 & 673 & 846 & 23.29 \\
\hline Earth & 149.6 & 0.0 & 0.00 & 24085 & -10381 & 10657 & 27.84 \\
\hline
\end{tabular}

ii) It has the shortest transfer time (in the first revolution) of all orbits described. The period for an Earth-to-Earth trip is about 11 years and the legs connecting the Earth and the Lagrangian points $\mathrm{L}_{4}$ and $\mathrm{L}_{5}$ last about 1.8 years each way; iii) It has the closest approaches to the Lagrangian points visited (during the first and second revolutions).

Of course, maneuvers can be made to get any desirable result, like repetition of the first revolution only; repetition of the first two revolutions and so on. Several crossing points are available for a one-burn impulsive maneuver, if desirable. However, the most interesting application for the curious "swing-by" found in this trajectory is to build a "cycler transportation system" between the Earth and the Lagrangian points $\mathrm{L}_{4}$ and $\mathrm{L}_{5}$, as explained in the next section.

\section{A Cycler Transportation System Between the Earth and the Lagrangian Points $\mathrm{L}_{4}$ and $\mathrm{L}_{5}$}

The "swing-by" discovered in the previous section can be used to build a cycler transportation system between the Earth and the Lagrangian point $\mathrm{L}_{5}$. Suppose that the spacecraft starts at $\mathrm{L}_{5}$ with zero velocity. It is possible to apply an impulse of 0.0274 (816 $\mathrm{m} / \mathrm{s}$ ) such that its velocity goes to $\mathrm{V}_{\mathrm{x}}=-0.0271$ and $\mathrm{V}_{\mathrm{y}}=$ 0.0040. With this velocity, the spacecraft follows one trajectory that is part of the SHORT $-4-5$ trajectory, as shown in Fig. 6. Then, it goes to the Earth, makes the "swing-by" and returns to $\mathrm{L}_{5}$, arriving there with velocity $\mathrm{V}_{\mathrm{x}}=-0.0018, \mathrm{~V}_{\mathrm{y}}=0.0263$. At this point, it is possible to apply an impulse $\Delta \mathrm{V}=0.0337(1003.8 \mathrm{~m} / \mathrm{s})$, such that its velocity goes to $\mathrm{V}_{\mathrm{x}}=-0.0271, \mathrm{~V}_{\mathrm{y}}=0.0040$ again and it starts the cycler one more time. The time-line for a complete cycler is:

\begin{tabular}{|c|c|}
\hline $\mathrm{t}=0$ & $\begin{array}{l}\text { The spacecraft leaves } L_{5} \text { from rest (as seen } \\
\text { in the rotating frame) with an impulse of } \Delta V \\
=0.0274(816 \mathrm{~m} / \mathrm{s})\end{array}$ \\
\hline $\begin{array}{l}\mathrm{t}= \\
1.80 \\
\text { years }\end{array}$ & $\begin{array}{l}\text { The spacecraft arrives at the Earth, makes a } \\
\text { swing-by to reverse the sense of motion } \\
\text { and it starts going back to } L_{5}\end{array}$ \\
\hline $\begin{array}{l}\mathrm{t}= \\
7.62 \\
\text { years }\end{array}$ & $\begin{array}{l}\text { The spacecraft arrives at } \mathrm{L}_{5} . \mathrm{A} \text { new } \\
\text { impulse of } \Delta \mathrm{V}=0.0377(1003.8 \mathrm{~m} / \mathrm{s}) \text { is } \\
\text { applied to send it back to the Earth and to } \\
\text { start the cycler again }\end{array}$ \\
\hline
\end{tabular}




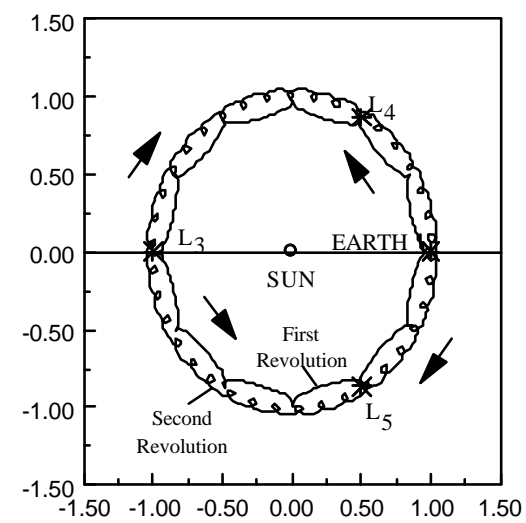

Fig. 5 - The Orbit "SHORT-4-5".

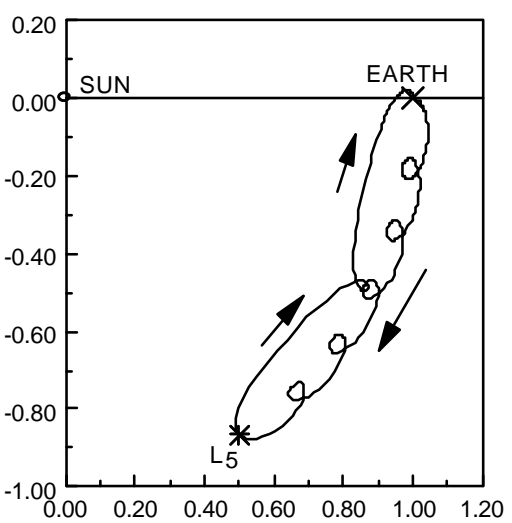

Fig. 6 - The Cycler System Between the Earth and $L_{5}$.

Another possibility to start the cycler again is to divide this last impulse in two parts. The first one has a magnitude of $\Delta \mathrm{V}=0.0264(786.1 \mathrm{~m} / \mathrm{s})$ and brings the spacecraft to a complete stop at $\mathrm{L}_{5}$. The second one is similar to the impulse applied in the beginning of the first revolution $(\Delta \mathrm{V}=0.0274=816 \mathrm{~m} / \mathrm{s})$ and it starts the next revolution of the cycler in the same way that it started the previous one. The advantage of this double impulse maneuver is that it is possible to keep the spacecraft parked at $\mathrm{L}_{\mathrm{g}}$ for as long as needed, allowing time to refuel, transfer of cargo, repairs, etc. The disadvantage is obviously the larger $\Delta \mathrm{V}$ required. The one-impulse maneuver requires $1003.8 \mathrm{~m} / \mathrm{s}$ and the two-impulse maneuver requires a total of $1602 \mathrm{~m} / \mathrm{s}$, that is $598.2 \mathrm{~m} / \mathrm{s}$ more expensive. To reproduce this cycler system for the Lagrangian point $\mathrm{L}_{4}$ there is no need for further calculations. By using the mirror image theorem it is only necessary to find the mirror image of the previous trajectory linking the Earth and the Lagrangian point $\mathrm{L}_{5}$. Note that the mirror image of the legs for an Earth-bound trip in now a $\mathrm{L}_{4}$-bound trip and the mirror image of the $\mathrm{L}_{5}$-bound leg is now the Earth-bound leg.

\section{The "LONG-5-4" Orbit}

In this orbit the spacecraft $\mathrm{M}_{3}$ leaves the Earth at $\mathrm{t}=0$ and goes to $\mathrm{L}_{5}$ (in 4.24 years), $\mathrm{L}_{3}$ (in 12.74 years), $\mathrm{L}_{4}$ (in 20.81 years) and back to the Earth's neighborhood again (in 24.88 years). The special properties of this orbit are:

i) This is the orbit with smaller residual velocity during the close approaches with the Lagrangian points among all the four orbits studied. This is important to: facilitate the data acquisition and/or a rendezvous with another spacecraft; reduce the magnitude of the impulse required to stop the spacecraft at the Lagrangian point;

ii) After completing the first revolution, the spacecraft makes a "swing-by" with the Earth, changes its direction of motion (as seen in the rotating frame) from "clock-wise" to "counter-clock-wise" and goes back to pass near $\mathrm{L}_{4}, \mathrm{~L}_{3}, \mathrm{~L}_{5}$ and the Earth, in a second revolution. The closest distance between the Lagrangian points and the spacecraft are a little bigger in the second revolution than in the first one, but a maneuver with a small $\Delta \mathrm{V}$ can provide closer approaches, if desirable. This orbit has the disadvantage of longer transfer times than the ones found in the "SHORT" versions, but it has the advantage of requiring smaller $\Delta \mathrm{Vs}$. The characteristic of changing its direction of motion can be used in the whole orbit, to make a complete tour to the points and start it again in the reverse order, or in part of it, to build a cycler transportation system between the Earth and the Lagrangian points $\mathrm{L}_{4}$ and $\mathrm{L}_{5}$, as done before. The details of this new version of this cycler system are explained better in the next section.

\section{An Option for a Faster Cycler Transportation System Between the Earth and $\mathrm{L}_{5}$ or $\mathrm{L}_{4}$}

The characteristic of reversing the direction of motion of the LONG-5-4 orbit can be used to build a new version of a system for permanent transportation between the Earth and the Lagrangian point $\mathrm{L}_{4}$. In this version, the spacecraft leaves $\mathrm{L}_{4}$ (by applying an impulse such that $V_{x}=26.8 \mathrm{~m} / \mathrm{s}$ and $V_{y}=47.7 \mathrm{~m} / \mathrm{s}$ ), goes to the Earth, and returns to $\mathrm{L}_{4}$ with the impulse given by the Earth's swing-by (with no necessity of fuel expenditure). Next, an extra impulse is applied, to make a fine adjustment that allows $\mathrm{M}_{3}$ to arrive at the Lagrangian point $\mathrm{I}_{4}$. Optimization techniques are not applied (although there is freedom to choose the 
position for the maneuver and the time of flight from this point to the destination point $\mathrm{L}_{4}$ ) to find the maneuver with minimum $\Delta \mathrm{V}$ for this case. A simple trial case (guessing a position for the impulse and a subsequent time of flight to $\mathrm{L}_{4}$ ) shows that an impulse of less than 0.02 canonical units (about $560 \mathrm{~m} / \mathrm{s}$ ) can satisfy all the requirements. Then, after $\mathrm{M}_{3}$ arrives at $\mathrm{L}_{4}$, it is necessary to apply another impulse to reverse its motion and send it back to the Earth, following the same trajectory it did in the first revolution. Again, a trial case that satisfies all the requirements, but without any optimization technique, shows that a maneuver with $\Delta \mathrm{V}$ less than 0.05 in canonical units (about $1500 \mathrm{~m} / \mathrm{s}$ ) is sufficient.

The final result is a trajectory that requires 4.0728 years for the Earth-bound trip, 1.7825 years for the $\mathrm{L}_{4}$ bound trip and about $2060 \mathrm{~m} / \mathrm{s}$ per revolution in maneuvers. It is a little mo re expensive than the previous cycler transportation system showed before $(2060 \times 1820$ $\mathrm{m} / \mathrm{s})$, but it is faster (5.86 $\times 7.62$ years). The decision for which trajectory to use depends on the specific requirements of the mission considered.

Again, a similar system can be build between the Earth and the Lagrangian point $\mathrm{I}_{3}$ by using the mirror image theorem, in the same way it was done before. The mirror image of the legs for an Earth-bound trip in now a $\mathrm{L}_{5}$-bound trip and the mirror image of the $\mathrm{L}_{4}$-bound leg is now the Earth-bound leg.

\section{$\underline{\text { Conclusions }}$}

In this paper, the problem of transferring a spacecraft from one body back to the same body in the planar restricted three-body problem is considered. Solutions are found for the Earth-Sun and Earth-Moon systems. Trajectories under this model with near-zero $\Delta \mathrm{V}$ to move a spacecraft between any two points on the group formed by the Earth and the Lagrangian points $\mathrm{L}_{3}$, $\mathrm{L}_{4}, \mathrm{~L}_{5}$ in the Earth-Sun system are found. It is shown how to apply these results to build a cycler transportation system to link all the points in this group. It is also shown how to use one or more "swing-by" with the Earth to build a cycler transportation system between the Earth and the Lagrangian points $\mathrm{L}_{4}$ and $\mathrm{L}_{5}$, with small $\Delta \mathrm{V}$ required for maneuvers in nominal operation.
The author wishes to express his thanks to CAPES (Federal Agency for Post-Graduate Education Brazil), INPE (National Institute for Space Research Brazil) and FAPESP (Foundation to Support Research in São Paulo State - Brazil) for supporting this research.

\section{$\underline{\text { References }}$}

1 Prado, A.F.B.A., 1993, "Optimal Transfer and Swing-By Orbits in the Two- and Three-Body Problems", Ph.D. Dissertation, University of Texas at Austin, TX-USA

2 Broucke, R.A. e Prado, A.F.B.A., 1993, "Jupiter SwingBy Trajectories Passing Near the Earth", Advances in the Astronautical Sciences, Vol. 82: Space Flight Mechanics, Part II, Editores: Robert G. Melton, Lincoln J. Wood, Roger C. Thompson, Stuart J. Kerridge, pp. 1159-1176.

3 Bender, D.F., 1979, "A Suggested Trajectory for a Venus-Sun, Earth-Sun Lagrange Points Mission, VELA," AAS paper 79-112. In: AAS/AIAA Astrodynamics Conference, Provincetown, MA, June 25-27.

4 Uphoff, C. and Crouch, M.A., 1991, "Lunar Cycler Orbits with Alternating Semi-Monthly Transfer Windows," AAS Paper 91-105. In: AAS/AIAA Spaceflight Mechanics Meeting, Houston, TX, February 11-13.

5 Aldrin, B., 1985, "Cyclic Trajectory Concepts," SAIC Presentation to the Interplanetary Rapid Transit meeting. Jet Propulsion Laboratory.

6 Szebehely, V.G., 1967, Theory of Orbits, Academic Press, New York.

7 Broucke, R.A. (1979), "Traveling Between the Lagrange Points and the Moon," Journal of Guidance, Control, and Dynamics, Vol. 2, No. 4, pp. 257-263.

8 Miele, A., 1960, "Theorem of Image Trajectories in the Earth-Moon Space," Astronautica Acta, pp. 225-232.

9 Hénon, M., 1968, "Sur les Orbites Interplanétaires qui Rencontrent Deux Fois la Terre," Bull. Astron., Vol. 3, pp. 377-402.

\section{Acknowledgments}

